\title{
IEEE Transactions on Instrumentation \& Measurement
}

\section{A novel actuating-sensing bone conduction-based system for active hand pose sensing and material densities evaluation through hand touch \\ --Manuscript Draft--}

\begin{tabular}{|c|c|}
\hline \multicolumn{2}{|l|}{ Manuscript Number: } \\
\hline Article Type: & Regular Article \\
\hline \multicolumn{2}{|l|}{ Section/Category: } \\
\hline Keywords: & $\begin{array}{l}\text { bone conduction; data gloves; accelerometers; measurement units; hand poses; } \\
\text { density estimation }\end{array}$ \\
\hline Corresponding Author: & $\begin{array}{l}\text { Giovanni Saggio } \\
\text { University of Rome } \\
\text { Rome, RM ITALY }\end{array}$ \\
\hline First Author: & Giovanni Saggio \\
\hline \multirow[t]{7}{*}{ Order of Authors: } & Giovanni Saggio \\
\hline & Angela Scioscia Santoro \\
\hline & Vito Errico \\
\hline & Maurizio Caon \\
\hline & Alfiero Leoni \\
\hline & Giuseppe Ferri \\
\hline & Vincenzo Stornelli \\
\hline Abstract: & $\begin{array}{l}\text { We realized and tested a novel system aimed at discriminating different hand poses by } \\
\text { means of an active actuating and sensing approach realized by converting } \\
\text { electromagnetic-to-mechanical waves (and vice-versa) and analyzing the } \\
\text { characteristics of the waves after their travel through the bones and cartilage of the } \\
\text { hand. The actuating part is realized through a transducer, placed on the dorsal part of } \\
\text { the hand, performing electromagnetic to mechanical energy conversion. The sensing } \\
\text { part is realized through an accelerometer, placed on a finger, performing mechanical to } \\
\text { electromagnetic energy conversion. } \\
\text { The way the sound propagates through the fingers mainly depend on the travelled } \\
\text { path, on the angles of fingers flexion, and on the (un)matched conditions with } \\
\text { surroundings. Therefore, the investigation of the characteristics of the returned signal } \\
\text { (in phase shifts and power spectral densities) can furnish information for discriminating } \\
\text { among different finger poses and different densities of touched materials. The system } \\
\text { highly performed in repeatability and reproducibility of the measures, well } \\
\text { discriminating among hand poses and among densities of touched materials. }\end{array}$ \\
\hline
\end{tabular}




\title{
A novel actuating-sensing bone conduction- based system for active hand pose sensing and material densities evaluation through hand touch
}

\author{
Giovanni Saggio, Angela Scioscia Santoro, Vito Errico, \\ Maurizio Caon, Alfiero Leoni, Giuseppe Ferri, Vincenzo Stornelli
}

\begin{abstract}
We realized and tested a novel system aimed at discriminating different hand poses by means of an active actuating and sensing approach realized by converting electromagnetic-to-mechanical waves (and vice-versa) and analyzing the characteristics of the waves after their travel through the bones and cartilage of the hand. The actuating part is realized through a transducer, placed on the dorsal part of the hand, performing electromagnetic to mechanical energy conversion. The sensing part is realized through an accelerometer, placed on a finger, performing mechanical to electromagnetic energy conversion.

The way the sound propagates through the fingers mainly depend on the travelled path, on the angles of fingers flexion, and on the (un)matched conditions with surroundings. Therefore, the investigation of the characteristics of the returned signal (in phase shifts and power spectral densities) can furnish information for discriminating among different finger poses and different densities of touched materials. The system highly performed in repeatability and reproducibility of the measures, well discriminating among hand poses and among densities of touched materials.
\end{abstract}

Index Terms - bone conduction, data gloves, accelerometers, measurement units, hand poses, density estimation.

\section{INTRODUCTION}

$\mathrm{D}$ URING the last decades, different technological approaches have been proposed to measure the hand capabilities and dexterities. Among them, probably the mainly addressed technology has been the sensory glove (also termed data, or goniometric glove), i.e. a glove hosting sensors capable to sense flexion/extension of the hand's fingers.

The sensory glove has been conveniently applied in different fields and for different purposes. In the medical field, it has

G.S., A.S.S., V.E. are with the Dept. of Electronic Engineering, University

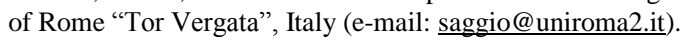

M.C. is with the University of Applied Sciences and Arts Western Switzerland (HES-SO). been used for assessing the functional recovery or improve rehabilitation effectiveness after hand surgery [1,2], and for evaluating surgeon's dexterity $[3,4,5]$. In the social field, it has been used for augmenting interaction possibilities of disabled people with machines [6] or prostheses [7], and for converting sign languages into written or oral sentences $[8,9]$. In the entertainment field, it has been used for controlling musical processes [10,11] even during live events [12], or for augmenting virtual reality interactions $[13,14]$. In work environments it has been used for recognizing hand visual signals [15], for remotely driving robotic hand [16,17], even in gravity-free conditions [18].

In spite of its undeniable usefulness, the sensory glove suffers from some disadvantages such as some wearing discomfort, loss of sense of touch and fine movement obstruction (mainly dependent on the fingertip cover, if any, and the type of cloth).

Therefore, it would be convenient to adopt a different approach for the measurement of the hand or fingers' capabilities and dexterities with a compact alternative solution capable of avoiding dressing a glove and wirings.

Within this frame, here we propose a bone conduction-based finger pose sensing approach aimed at different solution that could overcome the aforementioned disadvantages. Differently from the sensory glove, our system is not intended to measure each joints of each fingers, but the overall finger pose, useful for gesture recognition. In addition, we used the system to discriminate among materials with different densities, since when touched by the finger, the system responds differently.

\section{II.RELATED WORKS}

Bone conduction (BC) has been usually associated to the

A.L., G.F., V.S. are with the Dept. of industrial and Information Engineering and Economy, University of L'Aquila, Italy. 
auditory system [19], when acoustic waves stimulating the ear are converted into mechanical waves, in turn converted into neural waves by the cochlea [20], or when a BC headset allows hand-free and ear-free communication [21]. However, BC has becoming more and more generally associated to acoustic signals that propagates through the bones, for more generalized applications rather than the only auditory system [22,23,24,25].

Amento et al. realized a wristband with a piezoelectric microphone to recognize, through $\mathrm{BC}$, the sound produced by fingertip gestures such as tapping, rubbing, and flicking [26]. Deyle et al. made a wearable carrying case with two piezoelectric sensors to gather sounds occurring with wrist or foot gestures [27]. Harrison et al. localized finger tapping made on hand and arm by sensing with two arrays of five cantilevered piezo films [28]. Takemura et al. developed a bone-conduction based wearable sensing system to estimate the angle of an elbow joint [29]. Zhong et al. adopted the bone-conduction criteria for low-power non-intrusive low data-rate communication between implantable or wearable devices [30].

Here, we aimed at designing and testing a system made of an actuator, for electromagnetic to mechanical energy conversion, and a sensor, for returning to electromagnetic waves, in order to take advantage of $\mathrm{BC}$ for discriminating different finger poses and different materials when in full touch with the palm of the hand.

\section{III.MATERIALS AND METHOD}

\section{A.Actuating circuitry}

In order to realize electromagnetic to mechanical energy conversion, we adopted the surface transducer model COM10917 (by Sparkfun Electronics, US), working within 0.3-19 $\mathrm{kHz}$ frequency band, and equipped with a coil-pad system for the conversion.

An analog signal was generated by an Arduino MKR1000 and amplified by a class D audio digital amplifier, model PAM8403 (by Diodes Incorporated, US), for realizing a filtered signal (though a common-mode low-pass LC filter) capable to drive the $8 \Omega$ (@1 kHz) COM-10917 transducer load. The delivered $3.7 \mathrm{~V}$ sourced by a Li-Po battery was stabilized though voltage regulators (the U3V12F5 by Pololu Corporation, US, and the low drop voltage regulator LE35A by STMicroelectronics, Italy).

\section{B.Sensing circuitry}

The sensing part, for the mechanical to electromagnetic energy conversion, can generally be made through an accelerometer [31] or a microphone [32]. Our selection between technologies and among different device models took into account some requirements, such as the working frequency range, the signal-to-noise ratio, the detectable power spectral density, the power consumption, the dimensions, and the cost. In particular, as far as we know, no previous work was devoted at analyzing the most suitable working frequency for our specific application, so we realized a measurement set-up for sweeping among frequencies too. Therefore, for our verifications, we produced mechanical waves, by means of the

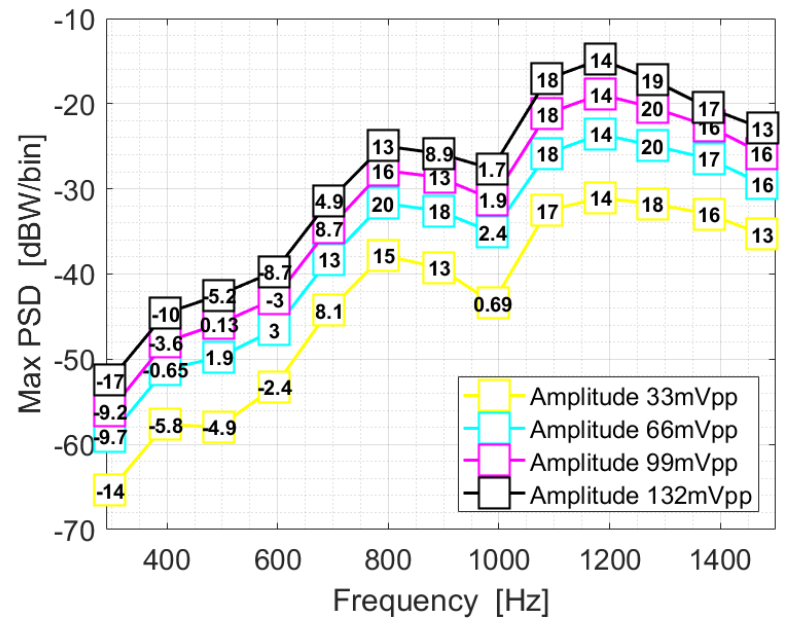

Fig. 1. Maximum PSD (and boxed SNR average values in $\mathrm{dB}$ ) vs. frequency using the BU-21771 accelerometer on suspended bone $(9 \mathrm{~cm}$ long and $0.8 \mathrm{~cm}$ in diameter) conditions, for different amplitudes of the testing sine signal.

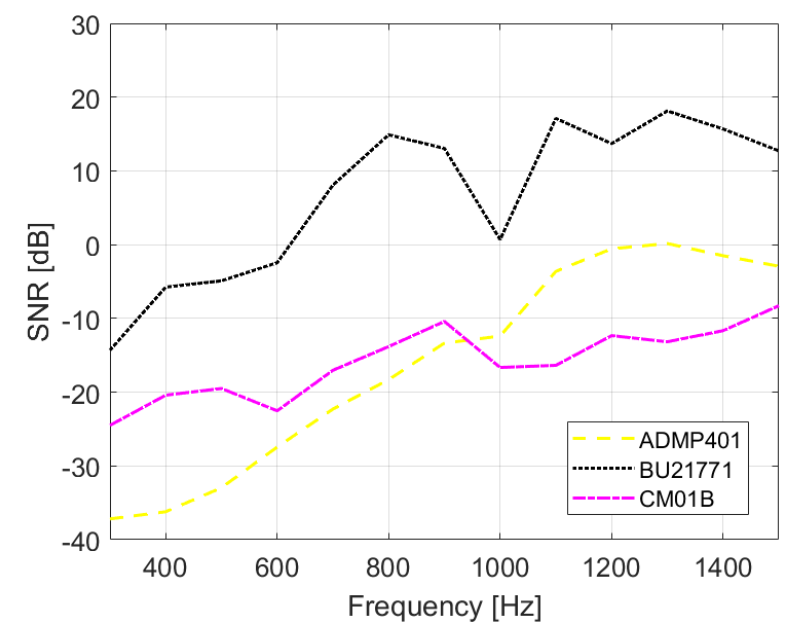

Fig. 2. SNR vs. frequency in the same conditions as Fig. 1, but comparing three different sensors, for amplitude of the testing sine signal equal to $33 m V_{p p}$.

surface COM-10917 transducer, previously tested on radius and ulna chicken bones $[33,34]$ different in length $(8.4,8.7,9.0,9.2$ $\mathrm{cm})$ and diameter $(0.4,0.8,0.8 \mathrm{~cm})$, when suspended in the air or stuck at the ends. The mechanical waves were induced by electric signals generated by Arduino, with frequencies ranging within $300-1500 \mathrm{~Hz}$ (stepped $100 \mathrm{~Hz}$ ) for testing the analog sensors, and frequencies ranging within $300-500 \mathrm{~Hz}$ (stepped $100 \mathrm{~Hz}$ ) for testing the digital sensor, while the peak-peak signal ranged within 33-132 mV (stepped $33 \mathrm{mV}$ ).

The mechanical waves were gathered by different sensors (in order to evidence the most suitable one for our purposes), which were two microphones and two accelerometers, in particular the analog microphone model CM-01B (by Measurement Specialties, US), the analog microphone model ADMP401 (by SparkFun Electronics, US), the analog accelerometer model BU-21771 (by Knowles Electronics, US), and the digital-output tri-axial accelerometer model ADXL345 (by SparkFun Electronics, US). The data of these sensors were stored by Arduino, forwarded via serial port to a personal computer, and analyzed through Matlab (by Matworks Inc., USA) routines. 
Data analysis allowed estimating the power spectral density (PSD) using the Welch method, and the signal to-noise-ratio (SNR). As a result, as a sensing device we selected the BU21771 sensor, because it outperformed the others with the highest SNR and PSD in most working conditions (Fig. 1 and Fig. 2), and also because of its small size, and the possibility to be interfaced to Arduino with two operational amplifiers only (rather than, for instance, the four amplifiers necessary to interface the CM-01B microphone).

The voltage supply requested by the BU21771 accelerometer was obtained reducing the 3.3 output voltage via Arduino by means of a buffered voltage divider. The output accelerometer signal was amplifier with the operational amplifier model MCP6231 (by Microchip Technology, US).

\section{C.Actuator testing frequency and placement}

Voltage sine waves sweeping within $300-1500 \mathrm{~Hz}$ in frequency and having a 600 peak-peak $\mathrm{mV}$ signal in amplitude, generated with a bench wave generator, fed the actuator for a conversion to mechanical waves, later gathered by the sensor by which we read PSD and SNR values obtained in correspondence of each finger. This operation was to determine the voltage sine frequency that allowed obtaining the highest PSD and SNR values but compatible with Arduino's signalsourcing characteristics with no data transfer rate degradation according to the UDP protocol. This testing protocol was repeated placing the actuator on the dorsal part of the hand both in parallel and orthogonal position with respect to the fingers.

According to the results, as optimal trade-off results we selected a testing frequency of $900 \mathrm{~Hz}, 132$ peak-peak $\mathrm{mV}$ in amplitude, with the actuator placed on hand dorsum (on the second metacarpus, centrally with respect to the thumb, index and middle finger) in parallel with the fingers (Fig. 3a, c).

\section{D.Densities tests}

To determine if and how the density of a generic material in contact with the hand affects the measurement, we performed a number of tests using 8 different homogeneous, in average isotropic, materials with the hand placed both without contact and with the palm in full contact with the material (Fig. $3 \mathrm{~b}$ and $3 c$, respectively). With generating sine voltage waves we measured the resulting PSDs, SNRs and the phase shifts gathered by the accelerometer.

\section{E.Hand posing tests}

We considered two opposite hand posing, such as a flat and a fist positions (Fig. 3b, d), so to evidence how, and to what extent, the actuating-sensing bone-conduction based system could evidence a discriminating capabilities between completely different hand configurations. In this case, for the data transmission between Arduino and a personal computer, we used serial communication first and then UDP communication.

However, since functional hand gestures mostly consist of grabbing gestures, we considered intermediate hand placements too, such as those with joints of the fingers not-completely flat-

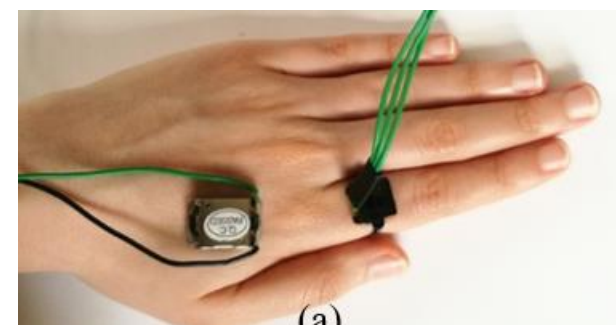

(a)

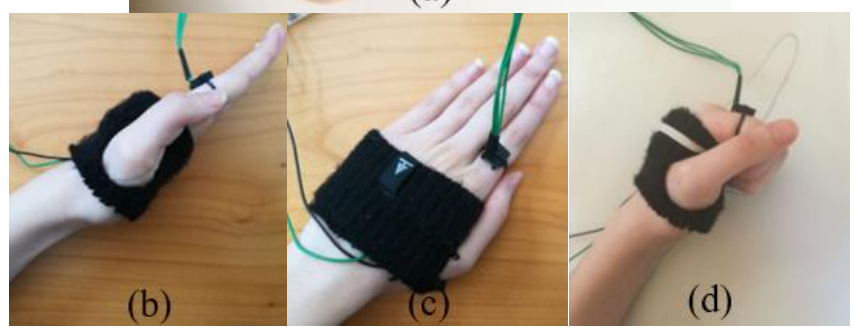

Fig. 3. (a) the actuator in parallel with respect to the fingers; the palm of the hand (b) without contact (flat hand) and (c) in contact with the material; (d) fist posing.

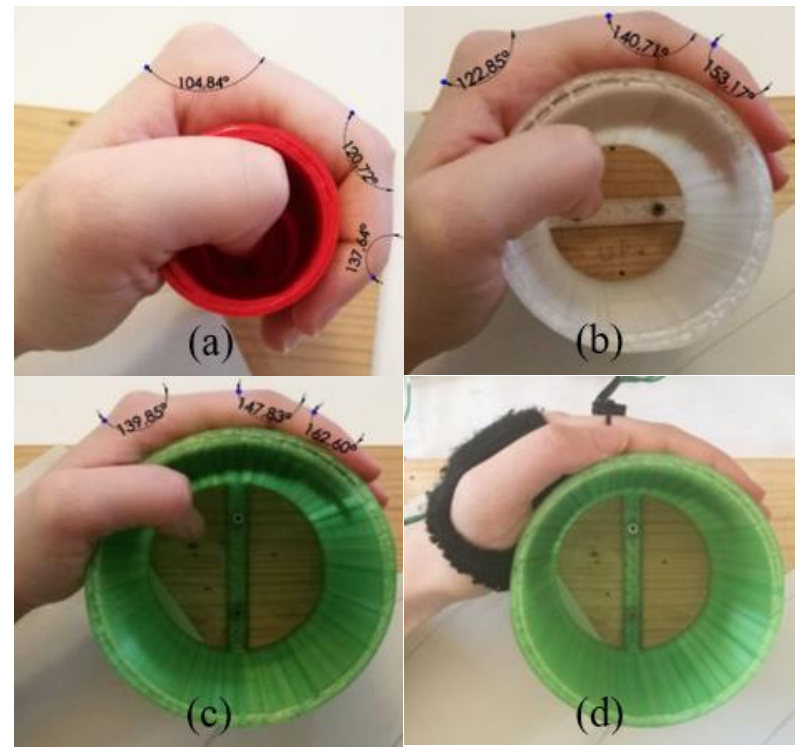

Fig. 4. Cylinder diameters and approximate bending angles for the MCP, PIP DIP index finger joints are (a) $4 \mathrm{~cm}, 105^{\circ}, 121^{\circ}, 138^{\circ}$; (b) $7 \mathrm{~cm}, 123^{\circ}, 141^{\circ}$, $153^{\circ}$; (c) $10 \mathrm{~cm}, 140^{\circ}, 148^{\circ}, 163^{\circ}$; respectively. The thumb is placed so to evidence the angles, but its position is not the used one for the measurements. (d) The correct thumb position during the tests.

or fist-flexed, as Fig. 4 shows. In this case, for the data transmission between Arduino and a personal computer, we used serial communication.

\section{F.Repeatability and reproducibility measurements}

In order to determine the reliability of our system, we evaluated repeatability and reproducibility of the measurements for hand posing by means of tests, from " $\mathrm{A}$ " to " $\mathrm{D}$ ", performed by a volunteer, respectively, as detailed in the following.

"Test A" for repeatability flat-fist posing: the volunteer was asked to keep the hand in a flat position for 6 secs, then to keep the hand in a fist position for 6 secs, flat + fist representing "one-trial" of measurements; "one-trial" was 10 times repeated obtaining "one-block" of measurements. "One-block" was 10 
times repeated pausing 2 minutes between blocks.

"Test B" for reproducibility flat-fist posing: the "test A" procedure was repeated but removing and replacing the actuating and sensing electronics on the hand between blocks.

"Test C" for repeatability grabbing posing: the "test A" procedure was performed except that grabbing around cylinders (one cylinder at time) was performed instead of flat-fist positions.

"Test D" for reproducibility grabbing posing: the "test B" procedure was performed except that grabbing around cylinders (one cylinder at time) was performed instead of flat-fist positions.

\section{IV.RESULTS AND COMMENTS}

The generated mechanical wave travels via bones and cartilages of the hand, the travel length, the attenuation, the phase shift depending, as a first approximation, on flexions of the fingers, and the amount of signal reflection depending, as a first approximation, on the (un)matched boundary conditions (i.e. hand in air or in contact with a material different in density). As a consequence, we can gather information about hand pose and density materials contacted by the hand (for a repeatable way of contact) considering phase shifts and PSD of the collected signals.

\section{A.Flat-fist hand grabbing test}

The actuating-sensing bone-conduction based system well performed in distinguish the hand when in flat or in fist posing (Fig. 3b, d), according to phase shift values obtained with respect to max PSD (Fig. 5a) and for both transmission protocols (serial and UDP) (Fig. 5b). In particular, the flat hand pose resulted with phase shift and max PSD within $80^{\circ}-85^{\circ}$ and $-68 \mathrm{dBW} / \mathrm{bin}$ and $-63 \mathrm{dBW} / \mathrm{bin}$, respectively, whilst the fist with phase shift ranging from $90^{\circ}$ to $95^{\circ}$ and max PSD ranging from $-76 \mathrm{dBW} /$ bin to $-68 \mathrm{dBW} /$ bin (Fig. 5a). With respect to the communication protocol, flat hand performed with phase shift lower than $85^{\circ}$ while fist resulted with phase shift higher than (around) $90^{\circ}$.

Also, the intermediate grabbing positions between flat and fist posing, with the joints of the fingers flexed with angles approximatively within 105 and 163 degrees (depending on the particular joint, see Fig. 6a, b, c, d) with hand wrapping a $4 \mathrm{~cm}$, $7 \mathrm{~cm}$ and $10 \mathrm{~cm}$ cylinder in diameter, respectively, were correctly distinguished with negligible errors, both for repeatability and reproducibility tests.

In fact, as Fig. 6a and b show, results from "test C" always evidenced a well distinguishable and repeatable signal phase shifts (around $93^{\circ}, 83^{\circ}, 71^{\circ}$ ) with a degree range of $2.66^{\circ}$ $\left(\mathrm{SD}=1.49^{\circ}\right), 7.78^{\circ}\left(\mathrm{SD}=1.68^{\circ}\right)$, and $6.38^{\circ}\left(\mathrm{SD}=1.94^{\circ}\right)$, for diameter of the cylinders of $4 \mathrm{~cm}, 7 \mathrm{~cm}$ and $10 \mathrm{~cm}$, respectively. As well, "test D" similarly performed with a reproducible signal phase shifts (around $91^{\circ}, 73^{\circ}, 83^{\circ}$ ) with slightly worst degree ranges, as it could be reasonably expected, of $4.72^{\circ}\left(\mathrm{SD}=1.2^{\circ}\right)$, $10.6^{\circ}\left(\mathrm{SD}=1.57^{\circ}\right)$, and $9.65^{\circ}\left(\mathrm{SD}=2.43^{\circ}\right)$, respectively.

Similarly, as Fig. $6 \mathrm{c}$ and $\mathrm{d}$ show, also the measured PSD values were demonstrated to be useful in discriminating grabbing poses. The repeatability assessed by "test C" resulted
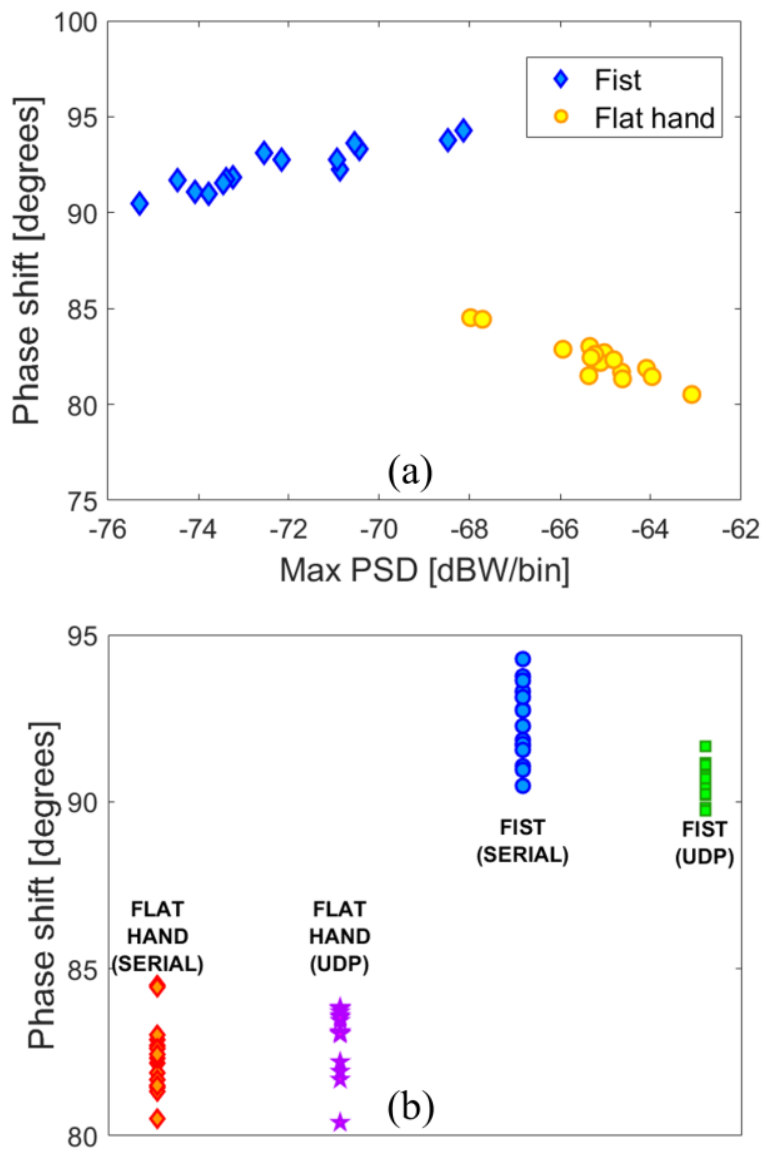

Fig. 5. (a) Phase shifts vs. max PSD for the hand in (•) flat and ( $\bullet$ ) fist placement (using serial communication); (b) Phase shift depending on the position (flat hand or fist) and the type of transfer used (serial communication or UDP communication).
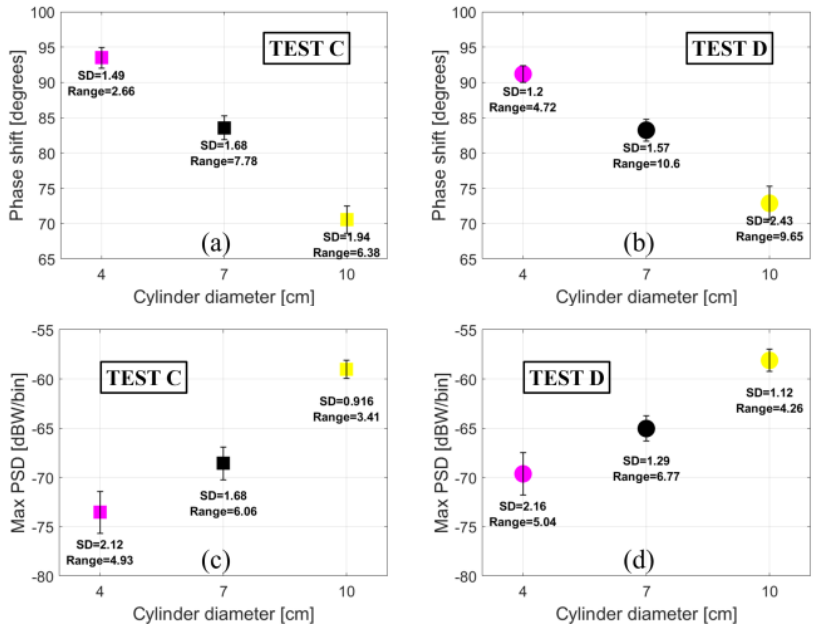

Fig. 6. (a), (b) Phase difference vs. cylinder diameter for "test C" and "test D", respectively; (c), (d) Max PSD vs cylinder diameter for "test C" and "test D", respectively. In each graph the mean standard deviation (SD) and the range (given by the difference between the maximum and the minimum average value obtained among the 10 averages relating to the 10 blocks) are reported. 
with ranges of $4.93^{\circ}\left(\mathrm{SD}=2.12^{\circ}\right), 6.06^{\circ}\left(\mathrm{SD}=1.68^{\circ}\right)$, and $3.41^{\circ}$ $\left(\mathrm{SD}=0.92^{\circ}\right)$ for diameters of cylinders of $4 \mathrm{~cm}, 7 \mathrm{~cm}$, and 10
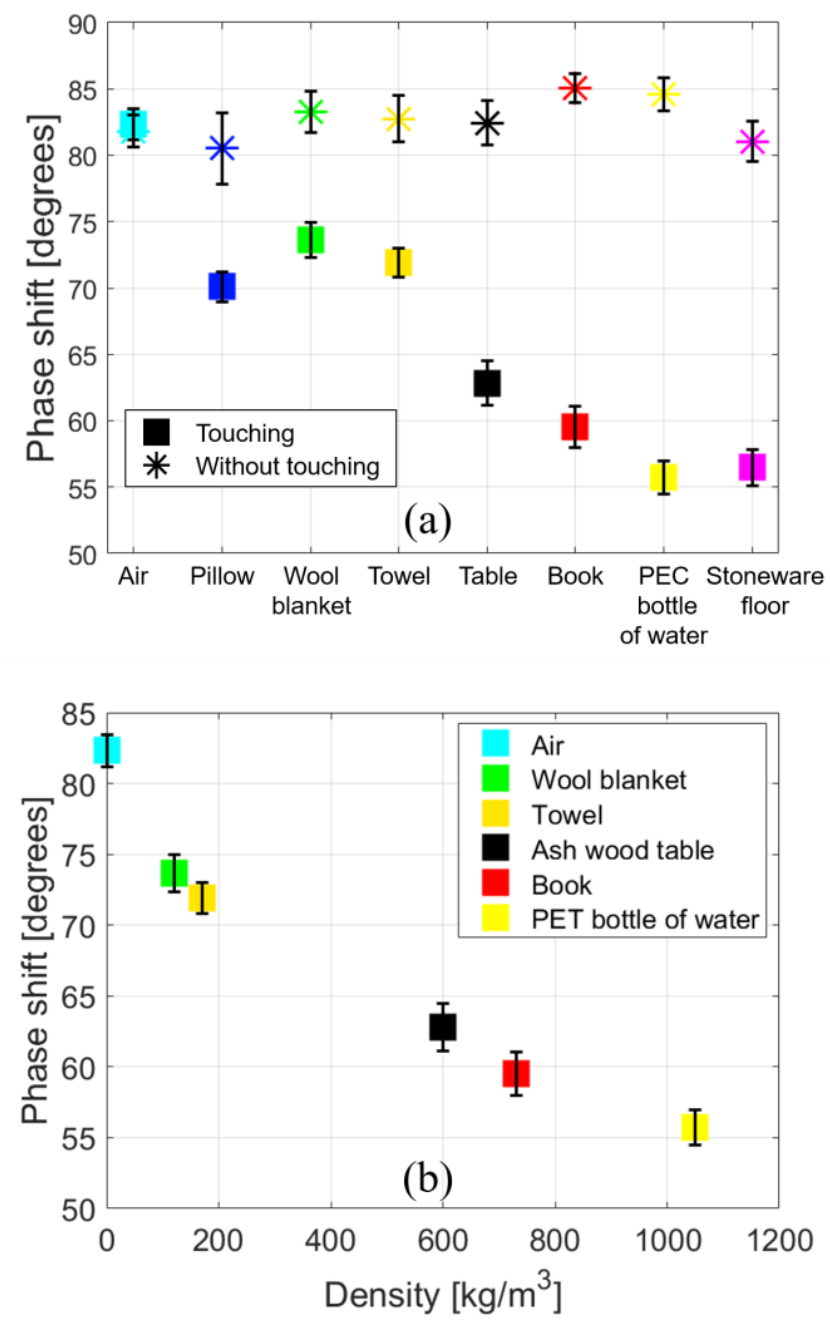

Fig. 7. (a) Phase shifts (in degrees) of the signal measured with the palm of hand touching (ם) and without touching (*) with different materials; (b) phase shifts (in degrees) of the signal measured with the palm of the hand touching different materials vs. the density of the touched material.

$\mathrm{cm}$, respectively; "test $\mathrm{D}$ " revealed a reproducibility of $5.04^{\circ}$ $\left(\mathrm{SD}=2.16^{\circ}\right), 6.77^{\circ}\left(\mathrm{SD}=1.29^{\circ}\right)$, and $4.26^{\circ}\left(\mathrm{SD}=1.12^{\circ}\right)$ again for the same diameters of the cylinders.

\section{B.Densities test}

For eight different materials (air, pillow, wool blanket, terry towel, ash wood table, book, plastic (PET) bottle with water, stoneware floor) and for two different hand positions (not touching and touching), the mean phase shift, with relative SD, were measured (Fig. 7a). When the hand does not touch any material, the average phase shifts always resulted within a small range $\left(80^{\circ}-85^{\circ}\right)$. Contrarily, with the palm of the hand touching different materials, the measured phase shift meaningfully changed (in a range of about $30^{\circ}, 55^{\circ}$ to $83^{\circ}$ ) of a different value for a different material. We estimated the six (out of the eight material densities as: plastic (PET) bottle with water $\simeq 1050$ $\mathrm{kg} / \mathrm{m}^{3}$, book $\simeq 730 \mathrm{~kg} / \mathrm{m}^{3}$, terry towel $\simeq 170 \mathrm{~kg} / \mathrm{m}^{3}$, wool blanket $\simeq 120 \mathrm{~kg} / \mathrm{m}^{3}$, ash wood table $\simeq 600 \mathrm{~kg} / \mathrm{m}^{3}$, and air $\simeq 1.3 \mathrm{~kg} / \mathrm{m}^{3}$. Fig. $7 \mathrm{~b}$ shows the measured phase shift with the palm of the hand touching different materials vs. the density of the touched material, with the result that the phase shift decreases with the density increasing.

\section{V.CONCLUSIONS}

We designed, developed, and validated a wireless wearable system for the hand, capable to distinguish different hand poses (flat, fist and three intermediate positions) and materials with different densities (air, pillow, wool blanket, terry towel, ash wood table, book, plastic (PET) bottle with water, stoneware floor) when touched.

The received signals differ from the generated ones in phase and in power spectral density depending on the hand pose or on the materials touched. This is because the wave signals are influenced by the material they went through (mainly bones and cartilages for the hand and the type of the material being touched) and the reflections they undergo (different with the amount of flexion of the fingers and with the hand-material mismatch).

Repeatability and reproducibility tests have confirmed that our system can be a valid alternative to those used to gather hand poses that are based on sensory gloves, on video analysis, or on electromyography.

\section{REFERENCES}

[1] G. Saggio, L. Sbernini, A. De Leo, M. Awaid, N. Di Lorenzo, A.L. Gaspari, A. L., "Assessment of hand rehabilitation after hand surgery by means of a sensory glove," in Proc. International Conference on Biomedical Electronics and Devices, Rome, Italy, 2016, pp. 187-194.

[2] H. Chen, Q. Wang, L. Cao, L. (2010, December). "Design of the workstation for hand rehabilitation based on data glove," in Proc. IEEE International Conference on Bioinformatics and Biomedicine Workshops (BIBMW), 2010, pp. 769-771.

[3] L. Sbernini, L.R. Quitadamo, F. Riillo, N. Di Lorenzo, A.L. Gaspari, G. Saggio, "Sensory-glove-based open surgery skill evaluation," IEEE Transactions on Human-Machine Systems, vol. 48, no. 2, pp. 213-218, 2018, 10.1109/THMS.2017.2776603

[4] G. Saggio, A. Lazzaro, L. Sbernini, F.M. Carrano, D. Passi, A. Corona, A., ... N. Di Lorenzo, "Objective surgical skill assessment: An initial experience by means of a sensory glove paving the way to open surgery simulation?," Journal of surgical education, vol. 72, no. 5, pp. 910-917, 2015, 10.1016/j.jsurg.2015.04.023

[5] F.M. Sánchez-Margallo, F. J. Pérez-Duarte, J.A. Sánchez-Margallo, M. Lucas-Hernández, A.M. Matos-Azevedo, I. Díaz-Güemes, “Application of a motion capture data glove for hand and wrist ergonomic analysis during laparoscopy," Minimally Invasive Therapy \& Allied Technologies, vol. 23, no. 6, pp. 350-356, 2014, 10.3109/13645706.2014.925928

[6] G. Saggio, S. Bocchetti, C.A. Pinto, G. Orengo, "Electronic Interface and Signal Conditioning Circuitry for Data Glove Systems Useful as 3D HMI Tools for Disabled Persons," in Proc. International Conference on Health Informatics (HEALTHINF), 2011, pp. 248-253.

[7] G. Orengo, L. Giovannini, G. Latessa, G. Saggio, F. Giannini, "Characterization of piezoresistive sensors for goniometric glove in hand prostheses," in Proc. 1st International Conference on Wireless Communication, Vehicular Technology, Information Theory and Aerospace \& Electronic Systems Technology, 2009, pp. 684-687.

[8] G. Saggio, P. Cavallo, A. Fabrizio, S.O. Ibe, "Gesture recognition through HITEG data glove to provide a new way of communication," in Proc. 4th International Symposium on Applied Sciences in Biomedical and Communication Technologies, 2011, pp. 1-5.

[9] G. Saggio, P. Cavallo, M. Ricci, V. Errico, J. Zea, M.E. Benalcázar, "Sign Language Recognition Using Wearable Electronics: 
Implementing k-Nearest Neighbors with Dynamic Time Warping and Convolutional Neural Network Algorithms," Sensors, vol. 20, no. 14, 3879, 2020, doi.org/10.3390/s20143879

[10] G. Saggio, F. Giannini, M. Todisco, G. Costantini, "A data glove based sensor interface to expressively control musical processes," in Proc. 4th IEEE International Workshop on Advances in Sensors and Interfaces (IWASI), 2011, pp. 192-195.

[11] E. McNutt, "Performing electroacoustic music: a wider view of interactivity," Organised Sound, vol. 8, no. 3, pp. 297-304, 2003, 10.1017/S135577180300027X

[12] G. Costantini, G. Saggio, M. Todisco, "A glove based adaptive sensor interface for live musical performances," in Proc. $1^{\text {st }}$ Int. Conf. on Sensor Device Technologies and Applications, 2010, pp. 217-220.

[13] J. Aleotti, S. Caselli, “A Desktop Virtual Reality System with Physical Animation and Glove Interaction," in Proc. Workshop on Virtual Reality Interaction and Physical Simulation (VRIPHYS), 2008, pp. 77-82.

[14] P. Kumar, J. Verma, S. Prasad, "Hand data glove: a wearable real-time device for human-computer interaction," Int. Journal of Advanced Science and Technology, vol. 43, pp. 15-25, 2012.

[15] G. Saggio, F. Cavrini, C.A. Pinto, "Recognition of arm-and-hand visual signals by means of SVM to increase aircraft security," in Proc. Int. Joint Conference on Computational Intelligence, 2015, pp. 444-461.

[16] B. Fang, D. Guo, F. Sun, H. Liu, Y. Wu, "A robotic hand-arm teleoperation system using human arm/hand with a novel data glove," in Proc. IEEE Int. Conference on Robotics and Biomimetics (ROBIO), 2015, pp. 2483-2488.

[17] A. Mishra, S. Malhotra, U. Pradesh, "Design of hand glove for wireless gesture control of robot," Int. J. Pure Appl. Math, vol. 114, pp. 69-79, 2017.

[18] G. Saggio, M. Bizzarri, "Feasibility of teleoperations with multi-fingered robotic hand for safe extravehicular manipulations," Aerospace Science and Technology, vol. 39, pp. 666-674, 2014, 10.1016/j.ast.2014.05.018

[19] M. Georgescu, A. Marinescu, A. Tonu, M. Radulescu, V. Budu, and M. Cernea, "Implantable bone-conduction hearing aid Baha system," $J$. Electr. Eng. Electron. Control Comput. Sci. JEEECCS, vol. 2, no. 3, pp. 53-60, 2016.

[20] P. Henry, T.R. Letowski, "Bone conduction: Anatomy, physiology, and communication (No. ARL-TR-4138)," Army research lab, 2007.

[21] Z. J. Lim, J. Claydon, "The use of bone conduction headsets to improve communication during the COVID- 19 pandemic," Emergency Medicine Australasia, under press, 2020, 10.1111/1742-6723.13611

[22] Y. Okawa, K. Takemura, "Haptic-enabled active bone-conducted sound sensing," in Proc. $28^{\text {th }}$ Annu. ACM Symp. User Interface Softw. Technol., 2015, pp. 87-88.

[23] C. Harrison, D. Tan, and D. Morris, "Skinput: Appropriating the body as an input surface," in Proc. Conf. Hum. Factors Comput. Syst., 2010, vol. 1, pp. 453-462.

[24] L. Zhong, D. El-Daye, B. Kaufman, N. Tobaoda, T. Mohamed, and M. Liebschner, "OsteoConduct: Wireless body-area communication based on bone conduction," in Proc. $2^{\text {nd }}$ Int. ICST Conf. Body Area Networks (BODYNETS), 2007, pp. 1-8.

[25] Y. Xin, T. Liu, H. Liu, L. Liu, Y. Li, T. Hou, ... \& S. Jin, “A Boneconduction Transducer Based Detection System for Sleep Apnea Screening in the Family Units," IEEE Sensors Journal, under press, 2020, 10.1109/JSEN.2020.3048310

[26] B. Amento, W. Hill, L. Terveen, "The sound of one hand: a wristmounted bio-acoustic fingertip gesture interface," in Proc. CHI'O2 Extended Abstracts on Human Factors in Computing Systems, 2002, pp. 724-725.

[27] T. Deyle, S. Palinko, E.S. Poole, T. Starner, T. "Hambone: A bioacoustic gesture interface," in Proc. 11th IEEE International Symposium on Wearable Computers, 2007, pp. 3-10.

[28] C. Harrison, D. Tan, D. Morris, "Skinput: appropriating the body as an input surface," in Proc. of the SIGCHI conference on human factors in computing systems, 2010, pp. 453-462.

[29] K. Takemura, A. Ito, J. Takamatsu, T. Ogasawara, "Active boneconducted sound sensing for wearable interfaces," in Proc. 24th annual ACM symposium adjunct on User interface software and technology, 2011, pp. 53-54.

[30] L. Zhong, D. El-Daye, B. Kaufman, N. Tobaoda, T. Mohamed, and M. Liebschner, "OsteoConduct: Wireless body-area communication based on bone conduction," in Proc. $2^{\text {nd }}$ Int. ICST Conf. Body Area Networks (BODYNETS), 2007, pp. 1-8.
[31] C. Zhang et al., "FingerPing: Recognizing fine-grained hand poses using active acoustic on-body sensing," in Proc. Conf. Hum. Factors Comput. Syst., 2018, pp. 1-10.

[32] B.C. You, S.C. Lo, C.K. Chan, H.L. Ho, S.C. Chiu, G.H. Hsieh, W. Fang, "Design and Implementation of a Novel Skull Vibration Sensing Module for Bone Conduction Microphone," in Proc. IEEE 33rd International Conference on Micro Electro Mechanical Systems (MEMS), 2020, pp. 118-121.

[33] P. H. F. Nicholson, P. Moilanen, T. Kärkkäinen, J. Timonen, and S. Cheng, "Guided ultrasonic waves in long bones: Modelling, experiment and in vivo application," Physiol. Meas., vol. 23, no. 4, pp. 755-768, 2002.

[34] V.C. Protopappas, D.I. Fotiadis, K.N. Malizos, "Guided ultrasound wave propagation in intact and healing long bones," Ultrasound Med. Biol., vol. 32, no. 5, pp. 693-708, 2006. 